\title{
Efficient 3D Morphable Face Model Fitting
}

\author{
Guosheng Hu ${ }^{\mathrm{a}, 1}$, Fei Yan ${ }^{\mathrm{a}}$, Josef Kittler ${ }^{\mathrm{a}}$, William Christmas ${ }^{\mathrm{a}, *}$, Chi Ho Chan ${ }^{\mathrm{a}}$, \\ Zhenhua Feng ${ }^{\mathrm{a}}$, Patrik Huber ${ }^{\mathrm{a}}$ \\ ${ }^{a}$ Centre for Vision, Speech and Signal Processing, University of Surrey, Guildford, GU2 7XH, UK
}

\begin{abstract}
$3 \mathrm{D}$ face reconstruction of shape and skin texture from a single $2 \mathrm{D}$ image can be performed using a 3D Morphable Model (3DMM) in an analysis-by-synthesis approach. However, performing this reconstruction (fitting) efficiently and accurately in a general imaging scenario is a challenge. Such a scenario would involve a perspective camera to describe the geometric projection from 3D to 2D, and the Phong model to characterise illumination. Under these imaging assumptions the reconstruction problem is nonlinear and, consequently, computationally very demanding. In this work, we present an efficient stepwise 3DMM-to-2D image-fitting procedure, which sequentially optimises the pose, shape, light direction, light strength and skin texture parameters in separate steps. By linearising each step of the fitting process we derive closed-form solutions for the recovery of the respective parameters, leading to efficient fitting. The proposed optimisation process involves all the pixels of the input image, rather than randomly selected subsets, which enhances the accuracy of the fitting. It is referred to as Efficient Stepwise Optimisation (ESO).

The proposed fitting strategy is evaluated using reconstruction error as a performance measure. In addition, we demonstrate its merits in the context of a 3D-assisted 2D face recognition system which detects landmarks automatically and extracts both holistic and local features using a 3DMM. This contrasts with most other methods which only report results that use manual face landmarking to initialise the fitting. Our method is tested on the public CMU-PIE and Multi-PIE face databases, as well
\end{abstract}

\footnotetext{
* Corresponding author: William Christmas

Email address: w. christmas a surrey.ac.uk (William Christmas)

${ }^{1}$ Current address: AnyVision, Queen's Road, Belfast, BT3 9DT, UK
} 
as one internal database. The experimental results show that the face reconstruction using ESO is significantly faster, and its accuracy is at least as good as that achieved by the existing 3DMM fitting algorithms. A face recognition system integrating ESO to provide a pose and illumination invariant solution compares favourably with other state-of-the-art methods. In particular, it outperforms deep learning methods when tested on the Multi-PIE database.

Keywords: face recognition; face reconstruction; 3D Morphable Model

\section{Introduction}

The intrinsic properties of 3D faces give scope for a representation that is immune to the kinds of variations in face appearance that are introduced by the imaging process such as viewpoint, lighting and occlusion. These invariant facial properties are potentially useful in a wide variety of applications in computer graphics and vision. However, recovering the 3D face and scene properties (viewpoint and illumination) from the appearance conveyed by a single 2D image is very challenging. Specifically, as noted in [1], it is impossible to distinguish between texture and illumination effects unless some assumptions are made to constrain them both. The 3D morphable face model (3DMM) [2] encapsulates prior knowledge about human faces that can be used for this purpose, and therefore potentially it is a good tool for 3D face reconstruction.

The 3DMM is a concise statistical model of a 3D face population created from 3D face data using principal component analysis (PCA). The model separately represents the face shape and surface texture. PCA removes data correlation and identifies a small number of latent variables which represent each face instance very efficiently. See also [3,4] for other developments of generative models applicable to 3D graph structures.

The reconstruction of a 3D face is conducted by a 3DMM fitting process, which estimates the 3D shape, texture, pose and illumination from a single 2D input image. Considerable research has been carried out to achieve efficient and accurate fitting. The methods advocated in the literature can be classified into two categories:

1. Simultaneous Optimisation (SimOpt): All the parameters (shape, texture, pose and illumination) are optimised simultaneously [2, 5, 6, 7]; 
2. Sequential Optimisation (SeqOpt): These parameters are optimised sequentially [8, 9, 10].

The SimOpt algorithms use gradient-based methods which are often slow and can easily get trapped in local minima. On the other hand, SeqOpt methods can have closed-form solutions for some or all of the parameters, and accordingly have the potential to be much more efficient computationally. However, the existing SeqOpt methods [8, 9, [10] make strong assumptions about the imaging camera and consequently they do not generalise well to faces distorted by perspective effects.

In this work we introduce a novel SeqOpt fitting framework, referred to as Efficient Stepwise Optimisation (ESO), which overcomes these problems and is an order of magnitude faster than existing methods. This framework groups the parameters to be optimised into 5 categories: camera model (pose), shape, light direction, light strength and albedo (skin texture). The fitting is decomposed into two separate processes: geometric fitting and photometric fitting.

Geometric Model Fitting Existing fast pose and shape fitting methods assume an affine camera model [9, 10] which is adequate provided the object's depth is small compared with its distance from the camera. A rule of thumb is that the object should be at least 10 times further from the camera than its depth. However, this is often not the case, e.g. when using a laptop camera for video conferencing, and for authentication, or when a camera is mounted on a vehicle windscreen for driver authentication, or monitoring the driver for tiredness. In such applications it is essential to relax the assumption and adopt a more general perspective camera model, which renders the reconstruction problem nonlinear, and consequently computationally expensive. In order to address this conundrum, we propose a novel approach to the shape fitting problem by formulating the fitting cost function in 3D, rather than the usual 2D. This formulation admits linearisation of the optimisation task which significantly enhances the computational efficiency.

As in [7] the occluding face contour is used to improve the shape fitting accuracy. In order to mitigate the additional processing costs, we propose to use landmarks on the occluding face contour (see Section 4.3 ) instead of face contour edges to refine 
the camera and shape estimates. To this end, we develop a method that automatically establishes the correspondence between the occluding contour landmarks of the input image and vertices of the 3D face model.

Photometric Model Fitting Both Phong [2, 7] and Spherical Harmonics models [9, 10] have been used in the past to estimate the illumination parameters. However, in order to model adequately both diffuse light and specularity, the latter method required many bases (81 in total) of spherical harmonics. Compared with the Spherical Harmonics approach, the Phong model has a more compact representation (elaborated further in Section 3), and is therefore used here. We found that it was adequate to model the illumination as a combination of a single distant point source plus uniform ambient light, thus keeping the number of coefficients to be found to a minimum.

To accelerate the light model fitting and skin texture parameter estimation, we present a novel approach to optimise both Phong model parameters and albedo. Specifically, we propose techniques (Section 4) to linearise the Phong model and the subsequent albedo estimation. Because the objective functions of these linear methods are convex, globally optimal solutions are guaranteed.

The measures to accelerate the illumination and texture reconstruction proposed in the paper speed up the fitting process by a factor of ten or more. We evaluate the fitting accuracy and show that it is superior to that achieved by the current alternatives. The impressive performance is the consequence of the ESO fitting process involving all the model vertices simultaneously, rather than just a randomly sampled subset.

We also evaluate the ESO fitting algorithm as part of a fully automatic pose- and illumination-invariant face recognition system. Its performance is at least comparable to the best performing state of the competitors, including solutions based on deep learning methods [11, 12] when evaluated on the Multi-PIE dataset.

The paper is organised as follows. In the next section we present a brief summary of the related work. The fitting problem is formulated in Section 3 to establish a methodological baseline. Our fast fitting algorithm ESO is developed in Section 4. The proposed algorithm is evaluated in Section 5 in terms of its reconstruction performance, as well as when embedded in a face recognition system. Section 6 draws the 


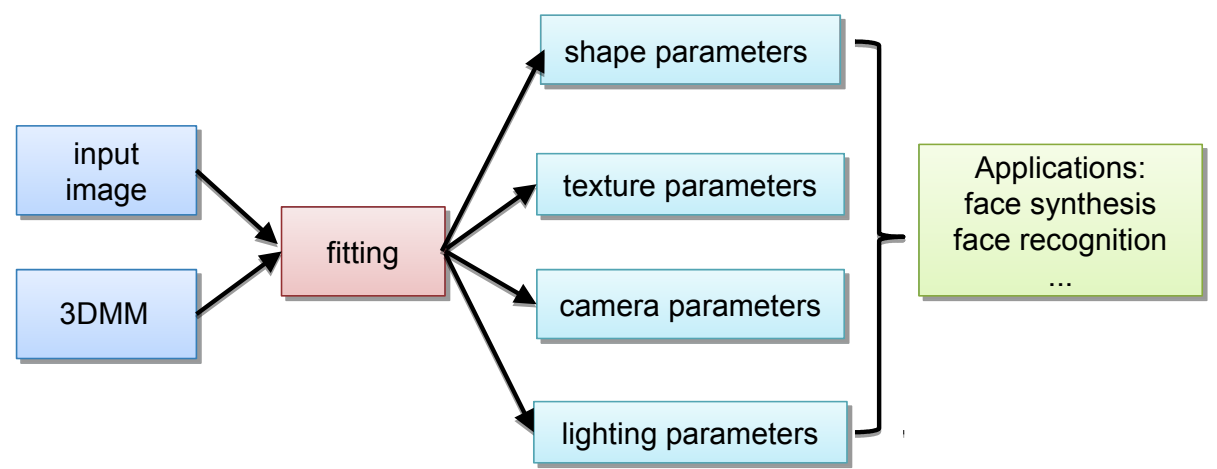

Figure 1: 3D morphable model fitting pipeline including the inputs and outputs of a fitting, and the applications of the fitting outputs.

paper to a conclusion.

\section{Related Work on 3D Morphable Model Fitting}

The 3DMM, first proposed by Blanz and Vetter [2], has successfully been applied to computer vision and graphics. A 3DMM consists of separate face shape and texture models learned from a set of 3D exemplar faces. These faces are represented as a graph, in which the node attributes are the 3D position and RGB colour at that node, and the edges indicate geometric connectivity. Related work (e.g. [13]) considers more complex image data. By virtue of a fitting process, a 3DMM can recover the face (shape and texture) and scene properties (illumination and camera model) from a single 2D image in a process schematically summarised in Fig. 1 The recovered parameters can be used for different applications, such as realistic face synthesis and face recognition. However, it is well known that achieving accurate fitting is particularly difficult for two reasons. Firstly, when recovering the 3D shape from a single 2D image, the 3D shape is generally projected to $2 \mathrm{D}$ in order to compare it with the $2 \mathrm{D}$ image features. As a result, the depth information of the 3D shape is lost. Secondly, separating the contributions of albedo and illumination is an ill-posed problem [14, 15]. Motivated by the above challenges, considerable research $[2,6,7,8,9,10]$ has been carried out to improve the fitting performance in terms of efficiency and accuracy. As mentioned 
in Section 1, these methods can be classified into two groups: SimOpt and SeqOpt.

In the SimOpt category, the fitting algorithm in [2, 5] minimises the sum of squared differences over all colour channels and all pixels between the input and reconstructed images. A Stochastic Newton Optimisation (SNO) technique is used to optimise a nonconvex cost function. Performance of this technique is poor in terms of both efficiency and accuracy because it is an iterative gradient-based optimiser which may end up in a local minimum.

The efficiency of optimisation is the driver behind the work of [6] where an Inverse Compositional Image Alignment algorithm [6] is introduced for fitting. The fitting is conducted by modifying the cost function so that its Jacobian matrix can be regarded as constant. In this way, the Jacobian matrix is precomputed, which greatly reduces the computational costs. However, this method cannot model illumination effects.

The Multi-Feature Fitting (MFF) strategy [7] is known to achieve the best fitting performance of the SimOpt methods. It makes use of many complementary features from an input image, such as edges and specularity highlights, to constrain the fitting process. The advantages of using these features are demonstrated in [7]. Further improvements to the MFF framework have been achieved by enhancing the fitting robustness to varying image resolution with a resolution-aware 3DMM [16], and by deploying a facial symmetry prior in [15] to ameliorate the quality of illumination fitting. However, all the MFF-based fitting methods are rather slow.

In the SeqOpt category, the 'linear shape and texture fitting algorithm' (LiST) [8] was proposed for improving fitting efficiency. The idea is to update the shape and texture parameters by solving linear systems. However, the illumination and camera parameters are optimised by the gradient-based Levenberg-Marquardt method, exhibiting many local minima. The experiments reported in [8] show that the fitting is of similar accuracy to the SNO algorithm, but much faster, in spite of the shape being recovered using a relatively slow optical flow algorithm. The drawback of this approach is the prerequisite that the light direction is known before fitting, which is not realistic for automatic analysis.

Another SeqOpt method [9] decomposes the fitting process into geometric and photometric parts. The camera model is optimised by the Levenberg-Marquardt method, 
and shape parameters are estimated by a closed-form solution. In contrast to the previous work, this method recovers 3D shape using only facial feature landmarks, and models illumination using spherical harmonics. Illumination and albedo are determined using least squares optimisation. The work in [17] improved the fitting performance of [9] by segmenting the 3D face model into different subregions. In addition, a Markov Random Field is used in [17] to model the spatial coherence of the face texture. However, the illumination models of [9, 17] cannot deal with specular reflectance because only 9 low-frequency spherical harmonics bases are used. In addition, [9, 17] use an affine camera model, which cannot model perspective effects.

In common with [9], two more recent SeqOpt methods [10, 18] also sequentially fit geometric and photometric models using least squares. Both methods use only facial landmarks to estimate pose and facial shape via an affine camera. They also share the use of spherical harmonics models to estimate illumination. The authors in [18] use 9 spherical harmonics bases, which cannot model specularity. The method in [10] can model specularity by projecting the RGB values of the model and input images to a specularity-free space for diffuse light and texture estimation. The specularity is then estimated in the original RGB colour space. In common with [9], both methods [10, [18] use an affine camera, which cannot model perspective effects. In addition, the colour of lighting in [10] is assumed to be known, which limits the applicability of the method.

Some works only focus on shape fitting [19, 20, 21]. In [20], around 100 facial landmarks are used to recover the facial shape employing the Levenberg-Marquardt algorithm as the optimiser. In contrast to [20], [19] uses local image features rather than facial landmarks as these features are more robust.

\section{3D Morphable face model and face image rendering}

A 3D face model is a representation of the surface of a class of objects - the objects in our case being faces. Each face consists of a set of vertices whose positions in 3D space collectively express the face shape. The vertices also each have an RGB pixel value, that collectively express the face skin texture (albedo). The model describes both 
the shape of a face and its appearance, determined by the surface texture. It is defined by a mesh of vertices $\mathcal{V}=\left\{v_{i} \mid i=1, \ldots, n\right\}$, sampling the face surface at a predefined set of facial points of semantic identity (eye corners, nose tip, etc). The $i$ th vertex $v_{i}$ of a face is located at $\mathbf{w}_{i}=\left(x_{i}, y_{i}, z_{i}\right)^{T}$, and has the RGB colour values $\left(r_{i}, g_{i}, b_{i}\right)$. Hence a $3 \mathrm{D}$ face is represented in terms of shape and texture as a pair of vectors:

$$
\mathbf{s}=\left(x_{1}, y_{1}, z_{1}, \ldots \ldots, x_{n}, y_{n}, z_{n}\right)^{T}, \mathbf{t}=\left(r_{1}, g_{1}, b_{1}, \ldots \ldots, r_{n}, g_{n}, b_{n}\right)^{T}
$$

Even for twins, faces are unique. Each individual will have a particular face shape and skin characteristics. The variability of face shape and skin texture in a population of individuals is captured by a statistical 3D face model, defined by a probability distribution in the $\mathbf{s}$ and $\mathbf{t}$ space. Since many vertex shape and texture measurements are highly correlated, a population of 3D faces inevitably lies in a subspace of the $\mathbf{s}$ and $\mathbf{t}$ space, typically determined by the Principal Component Analysis (PCA) or other sparse representation methods. Focusing on the former, let $\mathbf{S} \in \mathbb{R}^{3 n \times r_{s}}$ and $\mathbf{T} \in \mathbb{R}^{3 n \times r_{t}}$ denote the PCA bases of the $r_{s}$ shape and $r_{t}$ texture variations respectively. A face instance $(\mathbf{s}, \mathbf{t})$ can concisely be expressed as

$$
\mathbf{s}=\mathbf{s}_{0}+\mathbf{S} \boldsymbol{\alpha}, \quad \mathbf{t}=\mathbf{t}_{0}+\mathbf{T} \boldsymbol{\beta}
$$

where $\mathbf{s}_{0}$ and $\mathbf{t}_{0}$ are the mean face shape and texture respectively. The parameters $\boldsymbol{\alpha}$ and $\boldsymbol{\beta}$ are assumed to have normal distributions:

$$
\begin{aligned}
& p(\boldsymbol{\alpha}) \sim \mathcal{N}\left(0, \boldsymbol{\sigma}_{s}\right) \\
& p(\boldsymbol{\beta}) \sim \mathcal{N}\left(0, \boldsymbol{\sigma}_{t}\right)
\end{aligned}
$$

where $\sigma_{s}$ and $\sigma_{t}$ are the vectors of variances of the latent model shape and texture parameters.

As the bases and the mean vectors are fixed for a particular population, all statistical information is conveyed by the parameter vectors $\boldsymbol{\alpha}$ and $\boldsymbol{\beta}$, the dimensionality of which is considerably lower than that of the original face space. Each pair of model parameter vectors $\boldsymbol{\alpha}$ and $\boldsymbol{\beta}$ defines an instance of a 3D face. This provides a very concise representation of the face, which is convenient from the point of view of face synthesis. By changing the shape and texture parameters we can generate different faces. A 
transition from one pair of parameter vectors to another pair will morph one face to another in a smooth manner. This morphing capability of the statistical 3D face model has given it its name as the 3D Morphable Face Model (3DMM).

A 3DMM can be used for many purposes in face analysis. For instance, the model can be fitted to an input 2D face image, and the estimated shape and texture parameters of the reconstructed 3D face used for face recognition in the face model parameter space. Alternatively, given the pose of an input 2D face image, we can fit the 3DMM to a gallery face image and use the fitted 3D face to synthesise a new pose of the subject. For instance this could be a pose identical to the given pose in order to perform matching. Another possibility is to fit the 3DMM to an input 2D image of arbitrary pose, and then frontalise the query image with the help of the estimated 3D face shape. The operative phrase in all these use cases is $3 D M M$ fitting. It is the crucial prerequisite enabling all these applications.

The underlying principle of fitting a 3DMM to an input 2D face image is to identify the shape and texture parameters of the face model that would enable the synthesis of a 2D model image deemed indistinguishable from the input query image. However, the rendering process is quite complex. It involves not only the selection of model shape and texture parameters to produce a 3D face instance, but also its transformation to a new pose, and the subsequent projection of the $3 \mathrm{D}$ face to $2 \mathrm{D}$ under a particular scene illumination. The assessment of similarity of the synthesised image to the input image also traditionally involves sampling the input image at $2 \mathrm{D}$ points corresponding to the projection of the $3 \mathrm{D}$ mesh vertices onto the $2 \mathrm{D}$ input image.

Let us now describe the rendering process, the underlying physics of which is captured in Fig. 2 in more detail. We shall render a 2D view of a face instance showing a particular pose by rotating and translating the camera with respect to the face model coordinate system by a $(3 \times 3)$ rotation matrix $\mathbf{R}$ and a 3D translation vector $\boldsymbol{\tau}$ respectively. In the camera coordinate system the transformed shape $\mathbf{s}^{\prime}$ can be expressed in matrix form as

$$
\mathbf{s}^{\prime}=\mathbf{U} \mathbf{s}+\check{\tau}
$$

where $\mathbf{U}$ is the block diagonal matrix with $n$ copies of the rotation matrix $\mathbf{R}$ on its 
diagonal, and $\check{\tau}$ is a vector composed of $n$ copies of the displacement $\tau$.
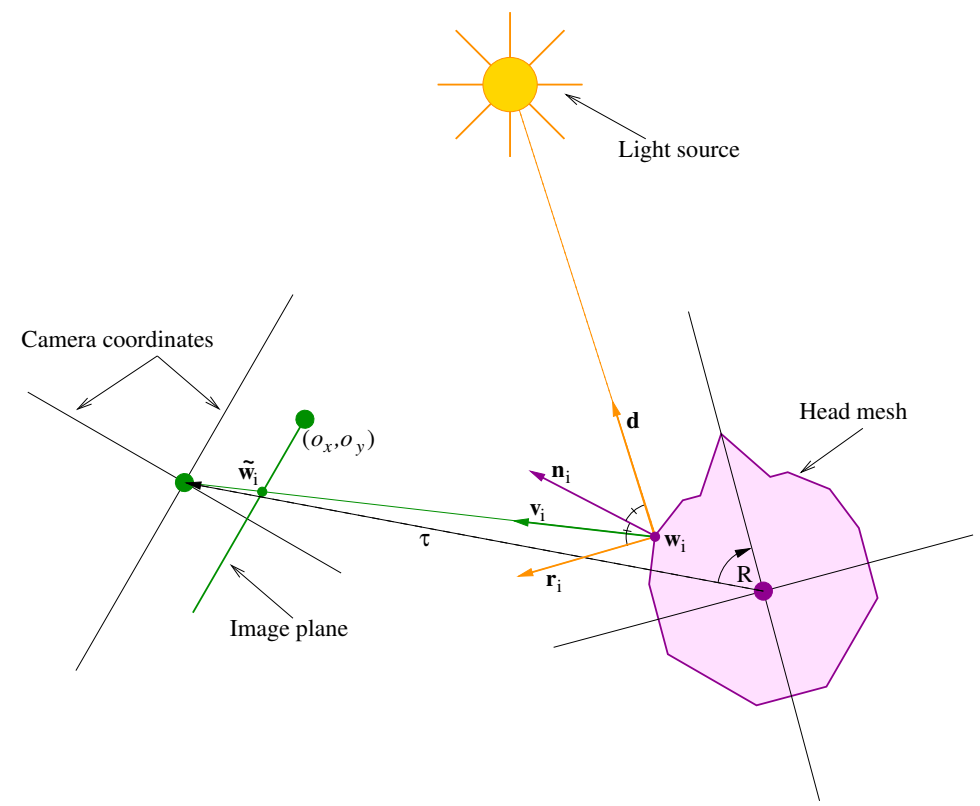

Figure 2: Physics of rendering: At image pixel position $\widetilde{\mathbf{w}}_{i}$, the RGB value output by the camera (small green blob) measures the reflection, on the face surface point $\mathbf{w}_{i}$, of the light source illuminating the face surface in direction $\mathbf{d}$. The surface normal at $\mathbf{w}_{i}$ is $\mathbf{n}_{i}$. In the head (purple blob) coordinate system, the camera is located at position $\boldsymbol{\tau}$ and the viewing direction of the vertex $\mathbf{w}_{i}$ is $\mathbf{v}_{i}$. The specular light is reflected from $\mathbf{w}_{i}$ centred on direction $\mathbf{r}_{i}$, where $\mathbf{r}_{i}$ is such that the surface normal $\mathbf{n}_{i}$ bisects $\mathbf{r}_{i}$ and the direction $\mathbf{d}$ of the incident light.

The pixel values at locations corresponding to $\mathbf{s}$ will depend on the albedo $t$ and the scene illumination. Different illumination models can be adopted for lighting the face (e.g. [22]), but we will adopt the Phong model which can represent complex reflectance phenomena, including specular reflectance, using a small number of parameters. The appearance of the generated face at each point, represented by a $3 n$-dimensional vector $\mathbf{a}^{M}$, is the product of the interplay of the face surface normal, skin albedo $\mathbf{t}$ and the incident light, assumed to be the sum of contributions from ambient, diffuse and specular lights:

$$
\mathbf{a}^{M}=\underbrace{\check{\mathbf{l}}_{a} * \mathbf{t}}_{\text {ambient }}+\underbrace{\left(\check{\mathbf{l}}_{d} * \mathbf{t}\right) *\left(\boldsymbol{N}_{3} \mathbf{d}\right)}_{\text {diffuse }}+\underbrace{\check{\mathbf{l}}_{d} * \mathbf{e}}_{\text {specular }}
$$

where the ambient light $\breve{\mathbf{l}}_{a}$ is a $3 n$-dimensional vector, composed of $n$ copies of the 
ambient light intensity $\mathbf{l}_{a}=\left(l_{a}^{r}, l_{a}^{g}, l_{a}^{b}\right)^{T}$ :

$$
\check{\mathbf{l}}_{a}=\left(l_{a}^{r}, l_{a}^{g}, l_{a}^{b}, \ldots \ldots . . l_{a}^{r}, l_{a}^{g}, l_{a}^{b}\right)^{T} \in \mathbb{R}^{3 n}
$$

Similarly, $\check{\mathrm{l}}_{d}$ is a $3 n$-dimensional vector, composed of $n$ copies of the directed light strength $\mathbf{l}_{d}=\left(l_{d}^{r}, l_{d}^{g}, l_{d}^{b}\right)^{T}$ :

$$
\check{\mathbf{l}}_{d}=\left(l_{d}^{r}, l_{d}^{g}, l_{d}^{b}, \ldots . ., l_{d}^{r}, l_{d}^{g}, l_{d}^{b}\right)^{T} \in \mathbb{R}^{3 n}
$$

The symbol $*$ denotes an element-wise multiplication operation. The matrix $\boldsymbol{N}_{3}$ is a stack of 3 copies of the matrices $\mathbf{N}$ :

$$
\mathbf{N}_{3}=\left[\mathbf{N}^{T}, \mathbf{N}^{T}, \mathbf{N}^{T}\right]^{T}
$$

where $\mathbf{N} \in \mathbb{R}^{n \times 3}$ is a stack of the surface normals $\mathbf{n}_{i} \in \mathbb{R}^{3}$ at vertices $i=1, \ldots, n$ (see Fig. 2). Unit vector $\mathbf{d} \in \mathbb{R}^{3}$ is the light direction. Vector $\mathbf{e} \in \mathbb{R}^{3 n}$ is a stack of the specular reflectance $\mathbf{e}_{i}$ of each vertex $i=1, \ldots ., n$ (the components of which could be different for the three channels), i.e.,

$$
\mathbf{e}_{i}=\mathbf{k}_{s}\left\langle\mathbf{v}_{i}, \mathbf{r}_{i}\right\rangle^{\gamma}
$$

where $\mathbf{v}_{i}$ is the viewing direction of the $i$ th vertex. Since in the face model coordinate system the camera is at position $\tau$, the viewing direction can be expressed as $\mathbf{v}_{i}=$ $\frac{\boldsymbol{\tau}-\mathbf{w}_{i}}{\left|\boldsymbol{\tau}-\mathbf{w}_{i}\right|}$ where $\mathbf{w}_{i}=\left(x_{i}, y_{i}, z_{i}\right)^{T}$ is the vector of the $3 \mathrm{D}$ coordinates of that vertex. Unit vector $\mathbf{r}_{i}$ denotes the reflection direction of the light source at the $i$ th vertex: $\mathbf{r}_{i}=2\left\langle\mathbf{n}_{i}, \mathbf{d}\right\rangle \mathbf{n}_{i}-\mathbf{d}$. The two constants $\mathbf{k}_{s}$ and $\gamma$ denote the specular reflectance and shininess respectively [23]. Note that $\mathbf{k}_{s}$ and $\gamma$ are determined by the facial skin reflectance property, which is similar for different people. They are assumed constant over the whole facial region. For the sake of simplicity, in our work, we also assume that $\mathbf{k}_{s}$ and $\gamma$ are the same for the three colour channels. Thus each entry in $\mathbf{e}_{i}$ is repeated three times. In this work, the components of $\mathbf{k}_{s}$ are each set to 0.175 , and $\gamma$ is set to 30 , following [23].

In all cases it is important to check all vertices for visibility so that parts of the face turned away from camera do not contribute to the rendered pixel values. 


\subsection{Fitting 3DMM to a $2 D$ image}

Let us consider an input face image $I$ acquired by a camera with focal length $f$ and the coordinates of its optical axis in the image plane $\mathbf{o}=\left(o_{x}, o_{y}\right)^{T}$. It is assumed that the image is landmarked. Let $\rho$ denote the set of extrinsic and intrinsic camera parameters

$$
\rho=\{\mathbf{R}, \boldsymbol{\tau}, f, \mathbf{o}\}
$$

and let us lump together all illumination parameters as

$$
\mu=\left\{\mathbf{l}_{a}, \mathbf{l}_{d}, \mathbf{d}, \mathbf{k}_{s}, \gamma\right\}
$$

Fitting the 3D face involves finding pose, shape, texture and illumination parameters $\rho, \boldsymbol{\alpha}, \boldsymbol{\beta}, \mu$ so that the image reconstructed from the model:

$$
\mathbf{a}^{M}=\left(r_{1}^{M}, g_{1}^{M}, b_{1}^{M}, \ldots \ldots ., r_{n}^{M}, g_{n}^{M}, b_{n}^{M}\right)^{T}
$$

is as close as possible to the input image.

Typically, the quality of the reconstruction is measured in 2D. This involves projecting the mesh of $3 \mathrm{D}$ vertices into $2 \mathrm{D}$. For each vertex the camera projects the triplet of its $3 \mathrm{D}$ coordinates into $2 D$ pixel location in the camera image plane as

$$
\tilde{\mathbf{s}}=\mathbf{P s}^{\prime}+\check{\mathbf{o}}
$$

where $\mathbf{P} \in \mathbb{R}^{2 n \times 3 n}$ is a block diagonal matrix constructed from the projection matrices $\mathbf{P}_{i}, i=1, \ldots n$ :

$$
\mathbf{P}_{i}=\left[\begin{array}{ccc}
\frac{f}{z_{i}^{\prime}} & 0 & 0 \\
0 & -\frac{f}{z_{i}^{\prime}} & 0
\end{array}\right]
$$

Note that each $\mathbf{P}_{i}$ is a function of the corresponding depth coordinate $z_{i}^{\prime}$, as well as the camera focal length $f$. The negative term in $\mathbf{P}_{i}$ results from an assumption of a clockwise image coordinate system. The $2 n$-dimensional vector o is a stack of $n$ copies of the $2 D$ position o of the optical axis in the image plane. For faces at a distance exceeding $10 \times$ the radius of a subject's head we can use an affine projection with $\mathbf{P}_{i}=\mathbf{P}_{j}, \forall i, \forall j$ instead, without incurring any significant approximation errors. 
The 2D mesh of projected vertices, $\tilde{\mathbf{s}}$, samples the input 2D image. Stacking the RGB values of the corresponding samples into a vector $\mathbf{a}^{I}$

$$
\mathbf{a}^{I}=\left(r_{1}^{I}, g_{1}^{I}, b_{1}^{I}, \ldots \ldots, r_{n}^{I}, g_{n}^{I}, b_{n}^{I}\right)^{T}
$$

we can then compare the synthesised and input images by measuring the error $\| \mathbf{a}^{I}-$ $\mathbf{a}^{M} \|$. Noting that the samples picked from the input image by the mesh are a function of $\rho$ and $\alpha$, the objective of the fitting process is to solve the optimisation problem

$$
\min _{\boldsymbol{\alpha}, \boldsymbol{\beta}, \rho, \mu}\left\|\mathbf{a}^{I}(\rho, \boldsymbol{\alpha})-\mathbf{a}^{M}(\rho, \boldsymbol{\alpha}, \boldsymbol{\beta}, \mu)\right\|^{2}+\lambda_{1}\left\|\boldsymbol{\alpha} \div \boldsymbol{\sigma}_{s}\right\|^{2}+\lambda_{2}\left\|\boldsymbol{\beta} \div \boldsymbol{\sigma}_{t}\right\|^{2}
$$

where the last two terms induce regularisation of the estimated parameters. The symbol $\div$ denotes element-wise division.

The problem formulated in 17 is very challenging because of its nonlinearity and its ill-posed nature [14, 15]. The conventional approach to optimisation is to apply the Newton Optimisation algorithm involving the sampling of random subsets of mesh vertices to achieve computational feasibility [2, 5]. These challenges motivated the developments reviewed in Section 2 but any speed-up therein is only achieved at the expense of restricted applicability.

In the following section we propose a novel method of fitting 3DMM that is more than an order of magnitude faster than the existing algorithms, without imposing any restrictions on the camera model and lighting. The computational efficiency is achieved by breaking the fitting problem up to create a sequence of optimisation tasks, most of which are linearised to render closed form solutions. The proposed strategy has the additional major benefit for illumination and albedo estimation of simultaneously involving all the model vertices in optimisation. This avoids local optima and leads to more accurate fitting.

\section{Efficient Stepwise Optimisation (ESO)}

This section describes our ESO framework. ESO is a SeqOpt method which groups all the parameters into 5 categories: pose with camera parameters, shape, light direction, light strength and albedo. The parameters in each group are optimised under the 


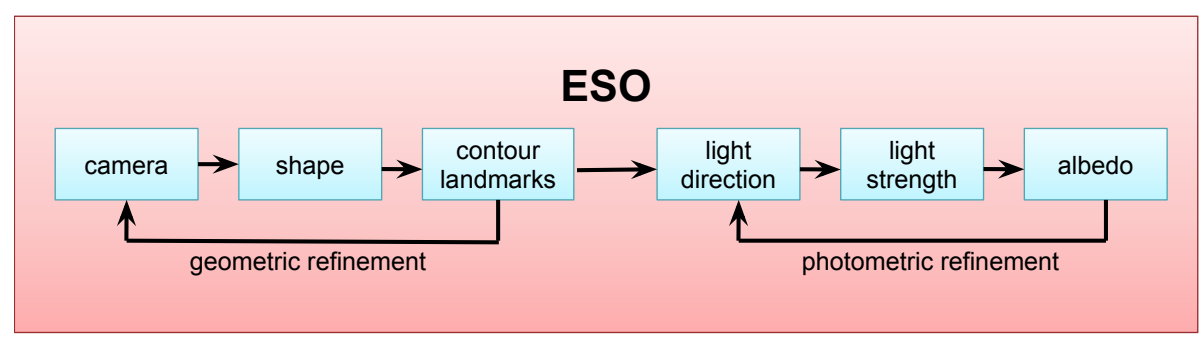

Figure 3: The ESO fitting process topology. Each of the two main phases of the fitting process - geometric and photometric - are iterated until convergence is achieved.

assumption that those in all the other groups are known, or have no impact on the optimisation process. The parameter grouping strategy aids the linearisation of the 3D face model fitting process, but further group-specific linearisation measures are adopted, as required. These are detailed in the respective sections.

The proposed method divides the fitting process into two phases, namely geometric and photometric optimisation as shown in Fig. 3. The geometric phase aligns an input image to a 3DMM, and the photometric phase recovers its reflectance. Each phase consists of three stages that are iterated in turn a few times to refine the solution. A key contribution of our approach is the proposed linearisation of all but one stage of the optimisation process that leads to closed-form solutions, and consequently computational efficiency. In Sections 4.1 to 4.6, each step of ESO is explained in more detail.

\subsection{Camera Parameter Estimation}

The first step uses the input image facial landmarks to estimate the subject's pose and the camera parameters that roughly align the input image to the model. Let us consider an identifiable point $\widetilde{\mathbf{w}}_{i}^{I}=\left(\tilde{x}_{i}^{I}, \tilde{y}_{i}^{I}\right)^{T}$ on the face of the input image, which semantically corresponds to the $i^{t h}$ vertex of the $3 \mathrm{D}$ face model with coordinates $\mathbf{w}_{i}=$ $\left(x_{i}, y_{i}, z_{i}\right)^{T}$. Image landmarks typically include the locations of the eye and mouth 


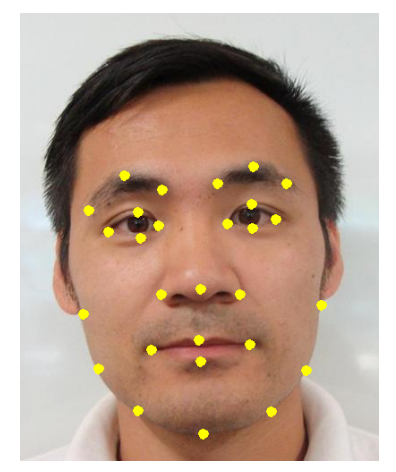

Figure 4: Visualisation of the facial landmarks used throughout this paper

corners, tip of the nose etc $2^{2}$ In this work, a maximum of 28 landmarks are used as shown in Fig. 4 However, some of these landmarks are not visible for non-frontal poses due to self-occlusion. In those cases, only the visible landmarks are used. Also, in the first iteration, the contour landmarks (7 shown in Fig 4 ) are not available.

For the alignment, we need to find the rigid transformation $\mathbf{R}, \boldsymbol{\tau}$ that moves the coordinates of the point $\mathbf{w}_{i}=\left(x_{i}, y_{i}, z_{i}\right)^{T}$ to its new position $\mathbf{w}_{i}^{\prime}=\left(x_{i}^{\prime}, y_{i}^{\prime}, z_{i}^{\prime}\right)^{T}$ so that after $\mathbf{w}_{i}$ is projected to $2 \mathrm{D}$ via the mapping $\mathcal{W}(\rho)$, its $2 \mathrm{D}$ coordinates $\widetilde{\mathbf{w}}_{i}=\left(\tilde{x}_{i}, \tilde{y}_{i}\right)^{T}$ are as close as possible to the image point $\widetilde{\mathbf{w}}_{i}^{I}=\left(\tilde{x}_{i}^{I}, \tilde{y}_{i}^{I}\right)^{T}$.

Let $\mathcal{L}$ denote the subset of vertices corresponding to the facial landmark points in the input image. Then the pose and camera parameters $\rho=\{\mathbf{R}, \boldsymbol{\tau}, f, \mathbf{o}\}$ can be estimated by minimising the distance between the input landmarks and those reconstructed from the model:

$$
\min _{\rho} \sum_{\forall i \in \mathcal{L}}\left\|\widetilde{\mathbf{w}}_{i}^{I}-\widetilde{\mathbf{w}}_{i}\right\|^{2}
$$

This is the only cost function which is not linearised. It is minimised by the LevenbergMarquardt algorithm [7], but because of the small number of parameters involved, the convergence is fast. Note that $\widetilde{\mathbf{w}}_{i}$ depends on both the pose and camera parameters, as well as the shape model $\mathbf{s}$. The latter is kept constant in this step, and in the first iteration, $\mathbf{s}$ is set to $\mathbf{s}_{0}$. In subsequent iterations, $\mathbf{s}$ is replaced by the shape update obtained

\footnotetext{
${ }^{2}$ Landmark detection itself is outside the scope of this paper.
} 
in the previous iteration by the second stage of the fitting process described in the next subsection. The estimated pose and camera parameters feed into the shape estimation stage described in Section 4.2 The contour landmarks described in Section 4.3 constrain the pose and camera parameters, and shape estimation.

\subsection{Shape Parameters Estimation}

Once the pose and camera parameters are recovered, the shape parameters $\alpha$ can be estimated. We linearise this problem by making use of the current estimate of the model vertex coordinates $z_{i}, \forall i$ to define the projection matrices $\mathbf{P}_{i}$. In addition, in contrast to prior art, we define the cost function in 3D space, as:

$$
\min _{\boldsymbol{\alpha}} \sum_{\forall i \in \mathcal{L}}\left\|\mathbf{w}_{i}^{I}-\mathbf{w}_{i}\right\|^{2}
$$

where the image landmarks $\widetilde{\mathbf{w}}_{i}^{I}, \forall i \in \mathcal{L}$, are back-projected to $\mathbf{w}_{i}^{I}=\left(x_{i}^{I}, y_{i}^{I}, z_{i}^{I}\right)^{T}$ via $\mathbf{w}_{i}^{I}=\mathcal{W}^{-1}\left(\widetilde{\mathbf{w}}_{i}^{I}, \rho\right)$. The main motivation for working in $3 \mathrm{D}$ is to reduce computational complexity further.

Since $\mathbf{w}_{i}$ is a vertex of the shape model $\mathbf{s}$, it is a function of $\boldsymbol{\alpha}$. The cost function is defined in 3D as:

$$
\min _{\boldsymbol{\alpha}}\left\|\hat{\mathbf{s}}^{I}-\hat{\mathbf{s}}(\boldsymbol{\alpha})\right\|^{2}+\lambda_{1} \boldsymbol{\alpha}^{T} \boldsymbol{\sigma}_{s}^{-1} \boldsymbol{\alpha}
$$

where: $\hat{\mathbf{s}}^{I}$ and $\hat{\mathbf{s}}$ are the stacked vertex positions $\mathbf{w}_{i}^{I}$ and $\mathbf{w}_{i}, \forall i \in \mathcal{L}$, respectively; $\hat{\mathbf{s}}=\hat{\mathbf{s}}_{0}+\hat{\mathbf{S}} \boldsymbol{\alpha} ; \hat{\mathbf{s}}_{0}$ and $\hat{\mathbf{S}}$ are constructed by choosing those elements from $\mathbf{s}_{0}$ and $\mathbf{S}$ (defined in Eq. (2)) corresponding to the landmark indices $\mathcal{L} ; \lambda_{1}$ is a free weighting parameter; $\boldsymbol{\alpha}^{T} \boldsymbol{\sigma}_{s}^{-1} \boldsymbol{\alpha}$ is a regularisation term based on Eq. 3 .

The closed-form solution for $\boldsymbol{\alpha}$ in is:

$$
\boldsymbol{\alpha}=\left(\hat{\mathbf{S}}^{T} \hat{\mathbf{S}}+\lambda_{1} \boldsymbol{\sigma}_{s}^{-1}\right)^{-1} \hat{\mathbf{S}}^{T}\left(\hat{\mathbf{s}}^{I}-\hat{\mathbf{s}}_{0}\right)
$$

where $\sigma_{s}$ is defined in Section 3

Finally, we explain how to implement the inverse projection $\mathcal{W}^{-1}$. Note that $\widetilde{\mathbf{w}}_{i}^{I}$ cannot be back-projected to $\mathbf{w}_{i}^{I}$ in the face model coordinate system unless $z_{i}^{I}$, the depth along the $z$ axis of $\mathbf{w}_{i}^{I}$, is known. Here, in the first iteration, $z_{i}^{I}$ is approximated 


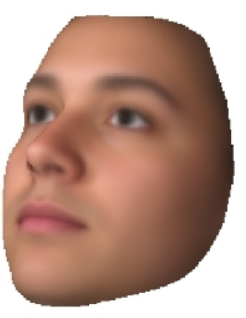

(a) model image

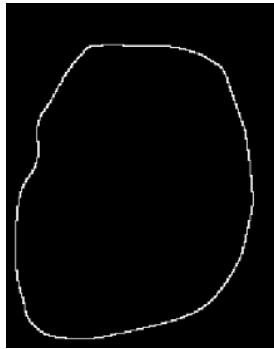

(b) contour edge

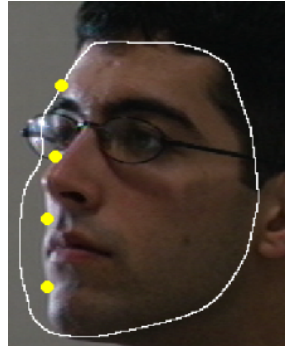

(c) image landmarks

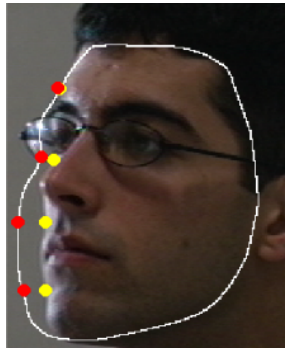

(d) correspondence

Figure 5: Contour landmarks detection. Yellow and red dots represent the contour landmarks of the input and model reconstructed images, respectively. Algorithm 1 bridges (c) and (d).

by the model vertex $z_{i}$, which is constructed from the mean shape $\mathbf{s}_{0}$. As the face shape is updated in subsequent iterations, the latest estimate $\mathbf{s}$ is used in place of $\mathbf{s}_{0}$.

\subsection{Contour Landmark Constraints}

One impediment to accurate 3D shape reconstruction from a non-frontal 2D face image stems from the lack of constraints on the projection of the occluding face contour. In [7], the authors define the contour edges as the occluding boundary between the face and non-face area, and use them to constrain the fitting. The contour edges of the 2D face image synthesised from a 3D model-based reconstruction of a 2D input image are shown in Fig. $5 \mathrm{~b}$. They are formed by linking the 3D model vertices lying on the occluding boundary of the projected 3D face mesh, determined by the vertex visibility check. A recent review of techniques developed to fit 3DMM to edges can be found in [24]. To reduce the computational cost of working with contour edges, we only use contour landmarks lying on the contour boundary. Here a contour landmark is defined as the point of intersection of the occluding boundary of a face and a horizontal line in the face coordinate system passing the corresponding symmetric landmark, as shown in Fig. 6. Such contour landmarks are labelled in the input image automatically by a cascaded-regression-based algorithm for automatic facial landmark detection [25], which has been trained to detect contour landmarks defined in this way.

The vertices $\mathcal{L}^{c}$ that form the contour landmarks along the occluding boundary 
of the fitted 3DMM are found using Algorithm 1. They are the vertices (red dots in Fig. 5d closest to the contour landmarks of the input image (yellow dots of Fig. 5c]. Once this correspondence is established, these contour landmark pairs are added to the available landmark set $\mathcal{L}$ in Eq. 18 and 20 to improve the estimation of camera parameters and shape.

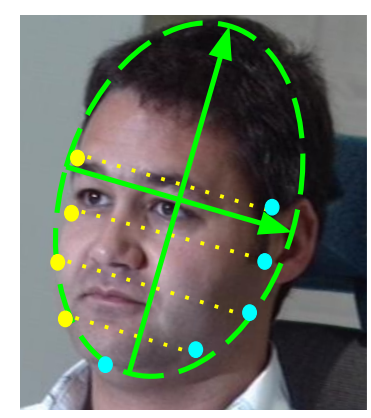

Figure 6: Definition of the contour landmarks. The axis of face symmetry is defined by the chin and the centre of the nose bridge. The face contour landmarks are the points of intersection of (i) the input image face occluding contour and (ii) the horizontal lines in the thus-defined face coordinate system, passing the visible facial contour landmarks.

\subsection{Light Direction Estimation}

After geometric fitting (Section 4.1 4.3), the 3DMM model is aligned to the input image, and the reflectance parameters can be estimated. In this step, we focus on the light direction d, and regard all other variables as constant. Recalling Eq. (6), the cost function can be formulated as:

$$
\min _{\mathbf{d}}\left\|\mathbf{a}^{I}-\check{\mathbf{l}}_{a} * \mathbf{t}-\left(\check{\mathbf{l}}_{d} * \mathbf{t}\right) *\left(\mathbf{N}_{3} \mathbf{d}\right)-\check{\mathbf{l}}_{d} * \mathbf{e}\right\|^{2}
$$

The minimisation of Eq. (22) is a non-linear problem because of the exponential form of $\mathbf{e}$ in Eq. (10). To eliminate this nonlinear dependence we precompute the value of $\mathbf{e}$ based on the assumptions that: $i) \mathbf{k}_{s}$ and $\gamma$ are constant; ii) the values of $\mathbf{v}$ and $\mathbf{r}$ are set to those of the previous iteration. In order to make the linearity of the light direction fitting problem more transparent, we avoid the element-wise multiplication 


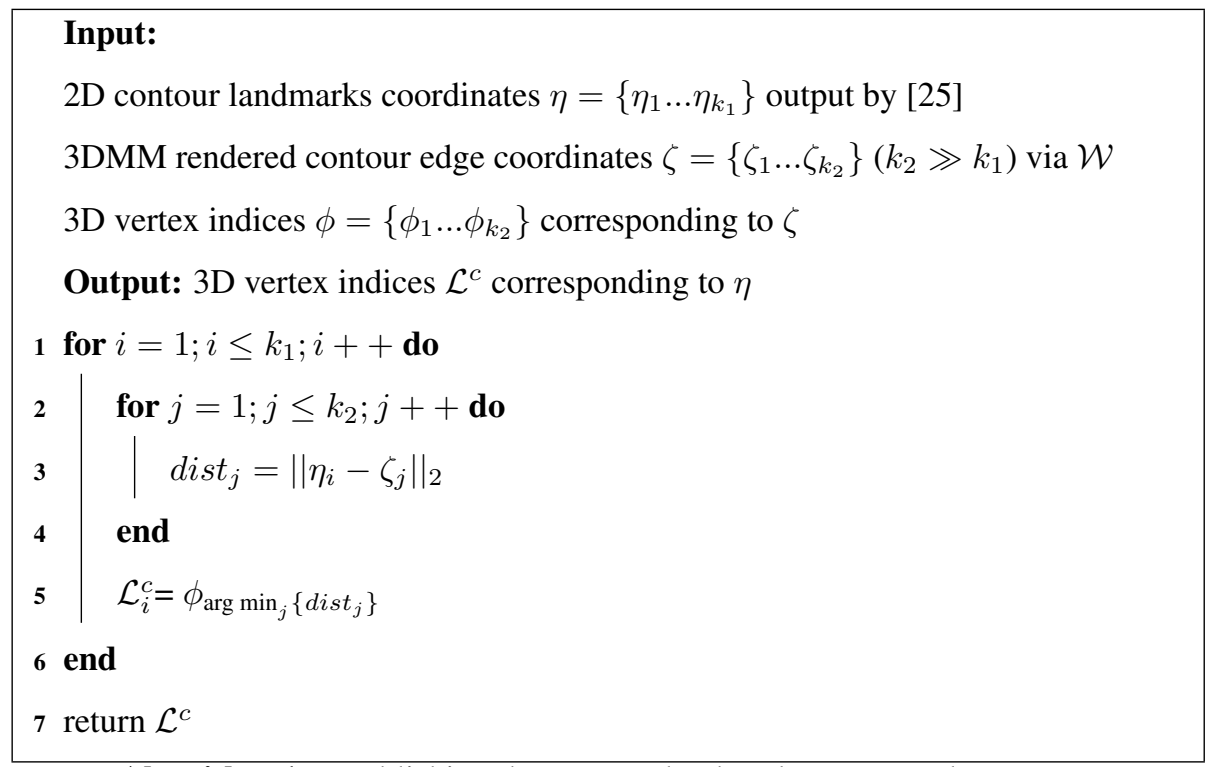

Algorithm 1: Establishing the contour landmark correspondence

in (22) by reformulating the cost function as:

$$
\min _{\mathbf{d}}\left\|\mathbf{a}^{I}-\check{\mathbf{l}}_{a} * \mathbf{t}-\check{\mathbf{l}}_{d} * \mathbf{e}-\left(\mathbf{A} * \mathbf{N}_{3}\right) \mathbf{d}\right\|^{2}
$$

where $\mathbf{A}=\left[\check{\mathbf{l}}_{d} * \mathbf{t}, \check{\mathbf{l}}_{d} * \mathbf{t}, \check{\mathbf{l}}_{d} * \mathbf{t}\right] \in \mathbb{R}^{3 n \times 3}$. By this reformulation, a closed-form solution can be found as: $\mathbf{d}=\left(\left(\mathbf{A} * \mathbf{N}_{3}\right)^{T}\left(\mathbf{A} * \mathbf{N}_{3}\right)\right)^{-1}\left(\mathbf{A} * \mathbf{N}_{3}\right)^{T}\left(\mathbf{a}^{I}-\check{\mathbf{l}}_{a} * \mathbf{t}-\check{\mathbf{l}}_{d} * \mathbf{e}\right)$. Then $\mathbf{d}$ is normalised to a unit vector.

For the first iteration, we initialise the values of $\mathbf{t}, \check{\mathfrak{l}}_{a}$ and $\check{\mathrm{l}}_{d}$ as follows. 1) In common with [26, 27], we assume that the face is a Lambertian surface. Consequently, only the diffuse light in Eq. (6) is modelled. 2) The strengths of diffuse light $\check{\mathbf{l}}_{d}$ and albedo $\mathbf{t}$ are set to vectors whose entries are all 1 and $\mathbf{t}_{0}$ respectively. With these assumptions, the cost function in the first iteration becomes:

$$
\min _{\mathbf{d}}\left\|\mathbf{a}^{I}-\left(\mathbf{B} * \mathbf{N}_{3}\right) \mathbf{d}\right\|^{2}
$$

where $\mathbf{B}=\left[\mathbf{t}_{0}, \mathbf{t}_{0}, \mathbf{t}_{0}\right] \in \mathbb{R}^{3 n \times 3}$. The closed-form solution is: $\mathbf{d}=\left(\left(\mathbf{B} * \mathbf{N}_{3}\right)^{T}(\mathbf{B} *\right.$ $\left.\left.\mathbf{N}_{3}\right)\right)^{-1}\left(\mathbf{B} * \mathbf{N}_{3}\right)^{T} \mathbf{a}^{I}$.

The estimated light direction is fed into the light strength and albedo estimations detailed in Section 4.5 and Section 4.6 


\subsection{Light Strength Estimation}

Having obtained an estimate of $\mathbf{d}$, the ambient and directed light strengths can be recovered. Because the three colour channels can be processed independently, for simplicity only the red channel is described. The cost function for the red channel is:

$$
\min _{\mathbf{l}_{a d}^{r}}\left\|\mathbf{a}^{I, r}-\mathbf{C l}_{a d}^{r}\right\|^{2}
$$

where $\mathbf{a}^{I, r}$ is the red channel of $\mathbf{a}^{I} ; \mathbf{C}=\left[\mathbf{t}^{r}, \mathbf{t}^{r} *(\mathbf{N d})+\mathbf{e}^{r}\right] \in \mathbb{R}^{n \times 2}, \mathbf{t}^{r}$ and $\mathbf{e}^{r}$ are the red channels of $\mathbf{t}$ and $\mathbf{e} ; \mathbf{l}_{a d}^{r}=\left(l_{a}^{r}, l_{d}^{r}\right)^{T}$, where $l_{a}^{r}$ and $l_{d}^{r}$ are the strengths of ambient and directed lights of the red channel respectively. The closed-form solution for $\mathrm{l}_{a d}^{r}$ is:

$$
\mathbf{l}_{a d}^{r}=\left(\mathbf{C}^{T} \mathbf{C}\right)^{-1} \mathbf{C}^{T} \mathbf{a}^{I, r}
$$

Note that $\mathbf{t}$ is set as stated earlier in Section 4.4. The green and blue channels are solved in the same way.

\subsection{Albedo Estimation}

Once the light direction and strengths are recovered, the albedo can be estimated. To avoid over-fitting, we regularise the albedo estimation and generate the cost function:

$$
\min _{\boldsymbol{\beta}}\left\|\mathbf{a}^{I}-\left(\mathbf{t}_{0}+\mathbf{T} \boldsymbol{\beta}\right) * \check{\mathbf{l}}_{a}-\left(\mathbf{t}_{0}+\mathbf{T} \boldsymbol{\beta}\right) * \check{\mathbf{l}}_{d} *\left(\mathbf{N}_{3} \mathbf{d}\right)-\check{\mathbf{l}}_{d} * \mathbf{e}\right\|^{2}+\lambda_{2} \boldsymbol{\beta}^{T} \boldsymbol{\sigma}_{t}^{-1} \boldsymbol{\beta}
$$

where $\lambda_{2}$ is a free weighting parameter. The closed-form solution is

$$
\boldsymbol{\beta}=\left(\mathbf{T}^{T} \mathbf{T}+\lambda_{2} \boldsymbol{\sigma}_{t}^{-1}\right)^{-1} \mathbf{T}^{T}\left(\mathbf{a}_{i n}^{I}-\mathbf{t}_{0}\right)
$$

where $\sigma_{t}$ is as defined in Section 3 and $\mathbf{a}_{i n}^{I}$, the illumination-normalised image, is given by:

$$
\mathbf{a}_{i n}^{I}=\left(\mathbf{a}^{I}-\check{\mathbf{l}}_{d} * \mathbf{e}\right) \div\left(\check{\mathbf{l}}_{a}+\check{\mathbf{l}}_{d} *\left(\mathbf{N}_{3} \mathbf{d}\right)\right)
$$

where the symbol $\div$ denotes element-wise division as before. 


\subsection{Computational complexity}

The computational complexity of our method is dominated by the albedo estimation described above. From Eq. (28) we can see that, since $n \gg r_{t}$, the dominating computations are the two matrix multiplications, both of which have a complexity of $O\left(n r_{t}^{2}\right)$.

\section{Experiments}

In this section, a comprehensive evaluation of our methodology is described. First, face reconstruction performance is evaluated. Then, in face recognition experiments, we compare our ESO with the existing 3DMM methods and other state-of-the-art methods. We implemented two effective 3DMM fitting methods [7] and [10], and the free parameter settings of [7, 10] follow the original papers. The results of all the other methods are cited from their papers based on the same experimental settings.

\subsection{Face Reconstruction}

First, we present some qualitative fitting results in Fig. 7. These images are from the Multi-PIE database. The people in these images have different gender, ethnicity and facial features such as a beard and/or glasses. All these factors can cause difficulties for fitting. As can be seen in Fig. 7, the input images are well fitted. Note that our 3DMM does not model glasses. Therefore, the glasses of an input image, such as the 3rd person in Fig. 77, can confuse the fitting process. Despite it, our ESO reconstructs this face well, showing its robustness.

In order to quantitatively measure every component of ESO, the 2D input images and their corresponding ground truths of camera parameters, 3D shape, light direction and strength, and texture need to be known. To meet all these requirements, we generated a local database of rendered 2D images with all the 3D ground truth as follows: (1) We collected and registered 20 3D face scans. The first 10 scans are used for model selection, and the remaining scans are used for performance evaluation. (2) The registered 3D scans are projected to PCA space, parameterising the ground truth in terms of coefficients $\boldsymbol{\alpha}$ and $\boldsymbol{\beta}$. (3) Using the registered 3D scans, we rendered 2D images under 


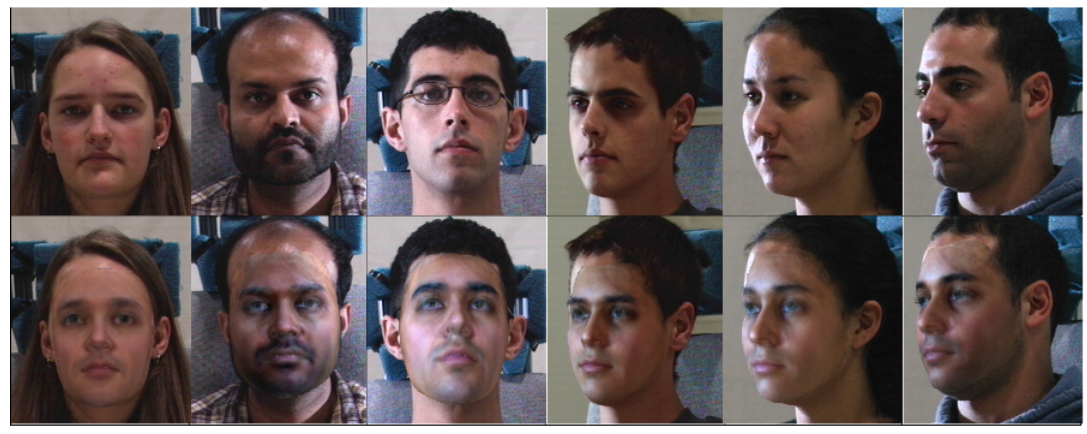

Figure 7: Row 1: input images with different pose and illumination variations. Row 2: ESOfitted/reconstructed images.

different poses and illuminations. (4) The 3DMM is fitted to obtain estimates of all these parameters. (5) Reconstruction performance is measured using cosine similarity between the estimated and ground-truth $\boldsymbol{\alpha}$ or $\boldsymbol{\beta}$.

\subsubsection{Effects of Hyperparameters}

Before we evaluate the face reconstruction performance, the sensitivity of the hyperparameters of ESO on the fitting process is investigated. The relevant hyperparameters are the regularisation weights $\lambda_{1}$ in Eq. 20) and $\lambda_{2}$ in Eq. 27) and the number of iterations $\left(l_{1}\right.$ and $\left.l_{2}\right)$ for geometric and photometric refinements (Fig. 3 , respectively. All the renderings in Section 5.1.1 are generated by setting both the focal length and the distance between the object and camera to 1800 pixels as suggested in [28].

Impact of the weight $\lambda_{1}$ on shape reconstruction The weight $\lambda_{1}$ should be selected carefully because improper $\lambda_{1}$ will cause under- or over-fitting during shape reconstruction. As shown in Fig. 8 , the reconstruction using a large $\lambda_{1}(=1000)$ looks very smooth and the shape details are lost, exhibiting typical characteristics of under-fitting. On the other hand, a small $\lambda_{1}(=0)$ causes over-fitting, and the reconstruction in Fig. 8 is excessively stretched. In comparison, the reconstruction with $\lambda_{1}=0.5$ recovers the shape well.

To quantitatively evaluate the impact of $\lambda_{1}, 2 \mathrm{D}$ renderings under 3 poses (frontal, side and profile), without directed light, are generated. To decouple the impact of $\lambda_{1}$ 


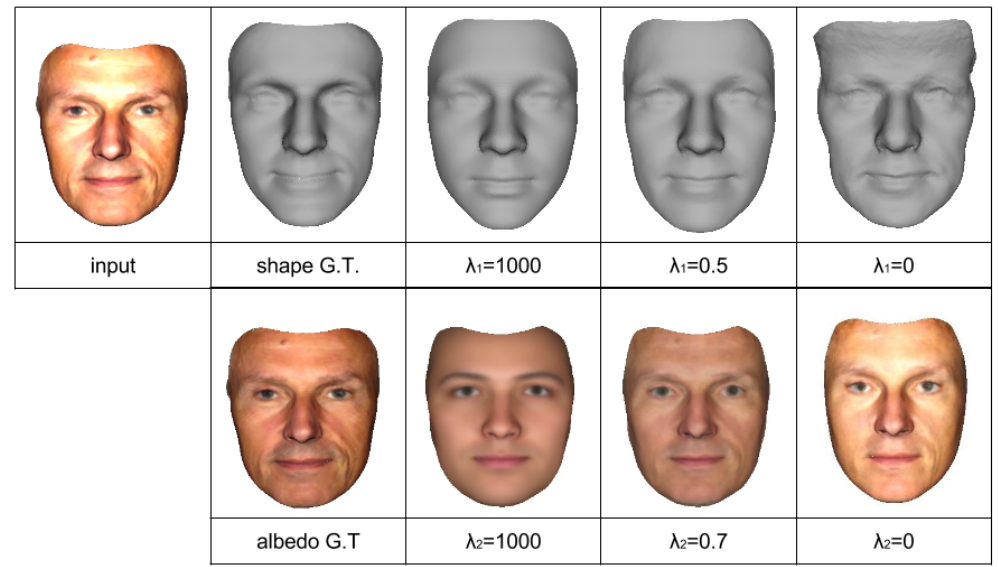

Figure 8: Impact of $\lambda_{1}$ and $\lambda_{2}$ on shape and albedo reconstruction. Column 1: input image, Column 2: ground truth of shape and albedo, Column 3-5: reconstructions with different $\lambda_{1}$ and $\lambda_{2}$.

and $l_{1}$ on shape refinement, $l_{1}$ is set to 1 . As shown in Fig. 9a neither small $(<0.4)$ nor large $(>1) \lambda_{1}$ lead to good reconstruction which is consistent with Fig. 8 . On the other hand, the reconstructions of all 3 poses do not change much with $\lambda_{1}$ in the region between 0.4 and 0.7 . Hence, $\lambda_{1}$ is set to 0.5 , which is the average value of the best $\lambda_{1}$ over all the test cases, to simplify parameter tuning.

Impact of the number of iterations $l_{1}$ on shape refinement The same renderings are also used to evaluate the sensitivity to $l_{1}$. From Fig. 9b, we can see that more than 3 iterations do not greatly improve the reconstruction performance for any pose. Therefore, $l_{1}$ is fixed at 3 in the remaining experiments.

Impact of the weight $\lambda_{2}$ on albedo reconstruction We also examine the impact of $\lambda_{2}$ on albedo reconstruction. Fig. 8 shows some qualitative results. Clearly, the reconstruction with $\lambda_{2}=1000$ loses the facial details because of being under-fitted. On the other hand, the one with $\lambda_{2}=0$ does not separate the illumination and albedo properly, causing over-fitting. In comparison, the one with $\lambda_{2}=0.7$ reconstructs the albedo well.

To quantitatively investigate the impact of $\lambda_{2}$ on the estimated light direction and 
strength, the renderings from different light direction $\mathbf{d}$ and strength $l_{d}^{3}$ are used as shown in Fig. 9c All these renderings are under frontal pose and $l_{2}=1$. It is clear that the reconstructed albedo does not change greatly with $\lambda_{2}$ in the region between 0.2 and 1. To simplify parameter tuning, $\lambda_{2}$ is fixed to 0.7 which is the average value of the best $\lambda_{2}$ over all the test cases.

Impact of the number of iterations $l_{2}$ on albedo refinement To investigate the impact of $l_{2}$, the same 2D renderings for the $\lambda_{2}$ evaluation are used. As shown in Fig. $9 \mathrm{~d}$, all the images converge by the 4 th iteration. Hence, for simplicity, $l_{2}$ is fixed to 4 in ESO.

\subsubsection{Reconstruction Results}

We evaluate shape and albedo reconstructions separately. ESO is compared with two methods: MFF [7] and [10], which are the best SimOpt and SeqOpt methods, respectively. We implemented the whole framework of MFF. Regarding [10], we only implemented the geometric (camera model and shape) part, because insufficient implementation details of the photometric part were released.

Shape Reconstruction As mentioned in Section 1 and 2 the affine camera used by [10] cannot model perspective effects, while the perspective camera used by ESO and MFF can. Different camera models lead to different shape reconstruction strategies. In order to find out how significant this difference is, we change the distance between the object and camera to generate perspective effects, at the same time keeping the facial image size constant by adjusting the focal length to match [28]. Note that the shorter this distance, the larger the perspective distortion. To compare shape reconstruction performance, 2D renderings under frontal pose obtained for 6 different distances are generated. We can see from Fig. 10 that the performance of ESO and MFF remains constant under different perspective distortions. However, the performance of [10] reduces greatly as the distance between the object and camera decreases. Also, ESO consistently works better than MFF under all perspective distortions.

Albedo Reconstruction We compare ESO with MFF [7] in Table 1] using images rendered under different light direction and strength. We see that the albedo reconstruction

\footnotetext{
${ }^{3}$ The illumination is set to be white here, i.e. $\mathbf{l}_{d}=\left(l_{d}, l_{d}, l_{d}\right)^{T}$
} 


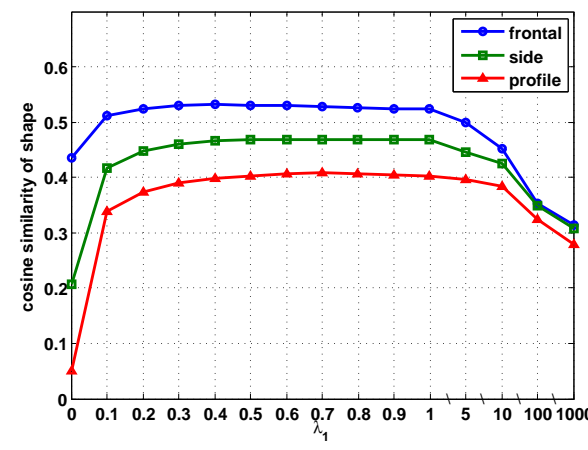

(a) Impact of regularisation weight $\lambda_{1}$ on shape reconstruction over poses

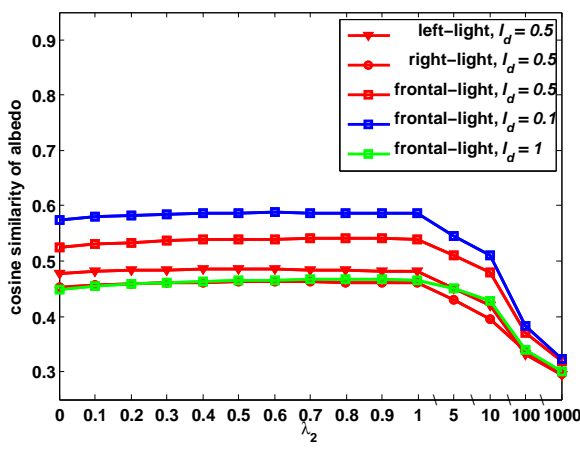

(c) Impact of regularisation weight $\lambda_{2}$ on albedo reconstruction over lightings

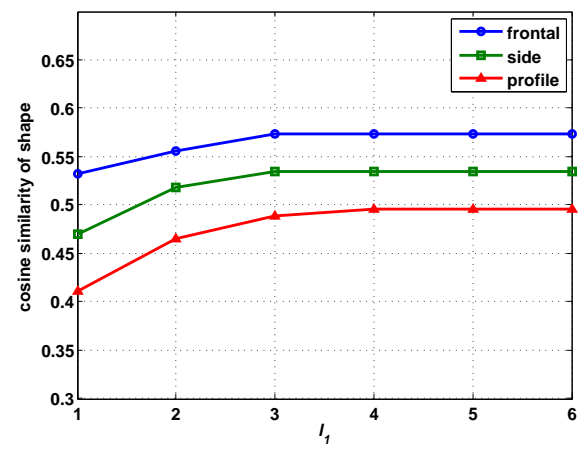

(b) Impact of the number of iterations $l_{1}$ on shape reconstruction

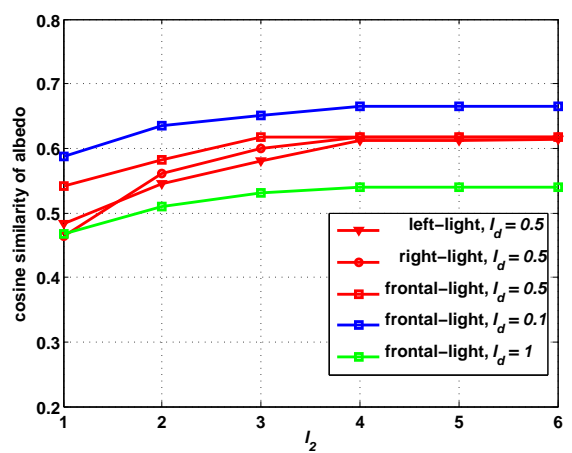

(d) Impact of the number of iterations $l_{2}$ on albedo reconstruction

Figure 9: Effects of hyperparameters on facial shape and albedo reconstruction

performance for different light direction is very similar, but it varies greatly for different directed light strength. This demonstrates that the albedo reconstruction is more sensitive to light strength than direction. Also, ESO consistently works better than MFF. The reasons are two fold: 1) MFF uses a gradient-based method that suffers from the non-convexity of the cost function. 2) For computational efficiency, MFF randomly samples only a small number (1000) of polygons to establish the cost function. This is insufficient to capture the information of the whole face, causing under-fitting. Our method being much faster makes use of all the polygons. Further computational 


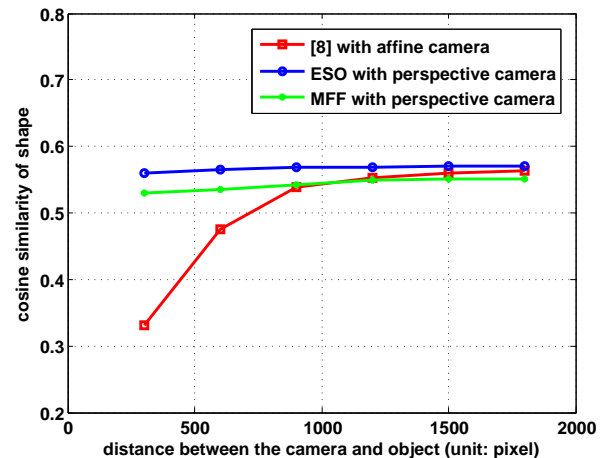

Figure 10: Shape reconstruction results measured by cosine similarity

Table 1: Albedo reconstruction results measured by cosine similarity.

\begin{tabular}{|c|c|c|c|}
\hline light direction & light strength $l_{d}$ & MFF [7] & ESO \\
\hline \hline left & 0.5 & $0.57 \pm 0.15$ & $\mathbf{0 . 6 1} \pm \mathbf{0 . 0 8}$ \\
\hline right & 0.5 & $0.57 \pm 0.13$ & $\mathbf{0 . 6 0} \pm \mathbf{0 . 0 8}$ \\
\hline frontal & 0.5 & $0.58 \pm 0.14$ & $\mathbf{0 . 6 2} \pm \mathbf{0 . 0 8}$ \\
\hline frontal & 0.1 & $0.60 \pm 0.13$ & $\mathbf{0 . 6 7} \pm \mathbf{0 . 0 7}$ \\
\hline frontal & 1.0 & $0.49 \pm 0.16$ & $\mathbf{0 . 5 4} \pm \mathbf{0 . 0 8}$ \\
\hline
\end{tabular}

efficiency discussions can be found in Section 5.2.1

\subsection{Pose and Illumination Invariant Face Recognition}

Pose- and illumination-invariant face recognition is a challenging problem addressed by a variety of approaches [29]. 2D methods address the pose and illumination problem at either pixel-level or image feature-level. The former aim to create pixel-level correspondence across different poses [30, 31, 32, 33]. For example, regression-based methods [31, 32] learn mapping matrices which project images of one particular pose to another one. The latter project the pixel values into pose- and/or illumination-robust feature spaces[11, 34, 35, 36, 37]. For example, Canonical Correlation Analysis [34] projects pixel values to a subspace where the impacts of pose and illumination are effectively removed. Deep Learning [11, 36] is also based on the same motivation.

3D methods intrinsically model pose variations using the analysis-by-synthesis approach. This means that a $3 \mathrm{D}$ face model has to be fitted to the input 2D image an- 
notated with facial landmarks. The methods can be categorised into 3 groups: pose normalisation [18, 38, 39], pose synthesis [40, 41] and 3D shape and texture feature extraction [2, 7, 42]. Pose normalisation renders all the images (gallery and probe) to a frontal view using 3D models; pose synthesis renders multiple gallery images of different poses for each subject. A probe image is only matched against that of the most similar pose in the gallery. 3D shape and texture feature extraction methods attempt to match probe and gallery images in a $3 \mathrm{D}$ parameter space, providing a pose- and illumination-invariant representation.

Among other options, 3DMM-based face recognition systems [7, 8, 9, 10] have a particular appeal because the process of $3 \mathrm{D}$ face model fitting provides a means of extracting the intrinsic 3D face shape and albedo from an unconstrained input face image. However, for a long time, the wide spread use of 3DMM in face recognition has been inhibited by inefficient 3D face model fitting algorithms. The ESO fitting algorithm presented in Section 4 offers a new means that greatly enhances the applicability of the 3D face model fitting approach.

Most existing 3DMM methods [7, 8, 9, 10] assume that accurate facial landmarks are known. To the best of our knowledge, only one previous work [43] proposes the use of automatically detected landmarks. In [43], the automatic landmark detection and 3DMM fitting are combined by a data-driven Markov chain Monte Carlo method. This method is robust to automatically detected landmarks but is rather slow. In contrast, we use an efficient cascaded regression technique [25] to automatically detect landmarks, which are then fed into a fully automatic face recognition system.

The conventional pipeline of a 3DMM face recognition system, shown as Scheme 1 in Fig. 11, involves the use of the generative 3D shape and texture parameters ( $\boldsymbol{\alpha}$ and $\boldsymbol{\beta})$, which are isolated from the input image appearance by suppressing the pose and illumination nuisance parameters. As in previous works [7, 9, 10], $\alpha$ and $\boldsymbol{\beta}$ are concatenated into a single vector to work as a holistic descriptor.

The drawback of holistic features is their inability to capture local facial properties, e.g. a scar, which may be very discriminative between people. To overcome this problem, we propose to extract local features as an alternative. Specifically, with the assistance of ESO fitting, we can render a pose- and illumination-normalised face image 


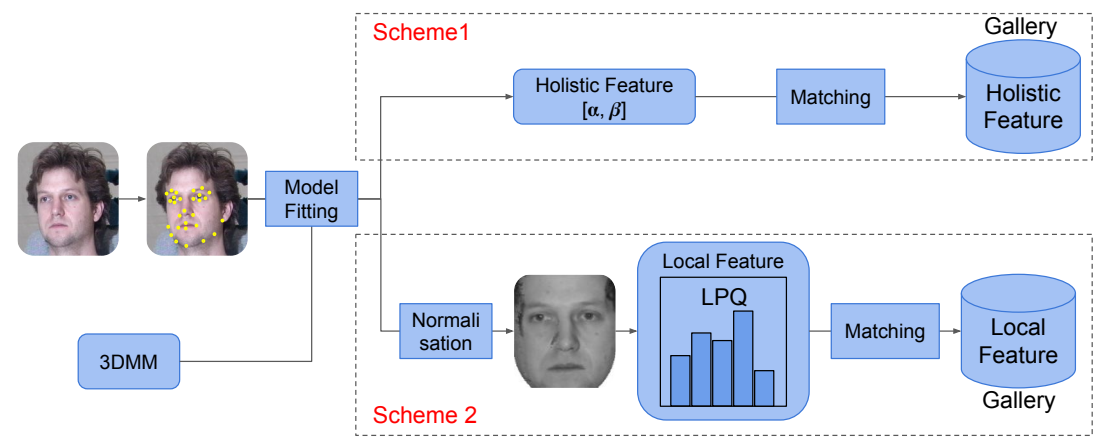

Figure 11: Face recognition pipeline. Scheme 1 and 2 use holistic and local features for face recognition respectively.

from an unconstrained input face as shown in Scheme 2 in Fig. 11. The pose normalisation is achieved by setting $\rho=\rho_{0}$ to transform the input face to a canonical frontal view. The illumination-normalised input image $\mathbf{a}_{i n}^{I}$ is obtained using Equation 29 Local features, such as those from the Local Phase Quantisation (LPQ) [44] descriptor used in this work, can then be extracted from this rendered image.

We evaluate the merit of the ESO fitting approach in the context of face recognition on the PIE [45] and Multi-PIE [46] databases which both have large pose and illumination variations. We set the hyperparameters $\left\{\lambda_{1}, l_{1}, \lambda_{2}, l_{2}\right\}$ of ESO to $\{0.5,3,0.7$, $4\}$ as discussed in Section 5.1

\subsubsection{PIE Database}

PIE is a benchmark database that can be used to compare different 3DMM fitting methods.

Protocol To compare all the methods fairly, the standard experimental protocol is used by our system. In particular, the recognition performance is measured using a subset of PIE including 3 poses (frontal, side and profile) and 24 illuminations. In order to conform to the protocol, in this experiment the fitting is initialised by manual landmarks. The gallery set contains frontal face images under neutral illumination, and the remaining images are probes. The holistic features $\boldsymbol{\alpha}, \boldsymbol{\beta}$ are used to represent a face.

Results Face recognition performance in the presence of combined pose and illumina- 
Table 2: Face recognition rate $(\%)$ on different poses averaging over all the illuminations on PIE

\begin{tabular}{|c|c|c|c|c|}
\hline & frontal & side & profile & average \\
\hline LiST [8] & 97 & 91 & 60 & 82.6 \\
\hline Zhang [9] & 96.5 & 94.6 & 78.7 & 89.9 \\
\hline Aldrian [10] & 99.5 & 95.1 & 70.4 & 88.3 \\
\hline MFF [7] & 98.9 & 96.1 & 75.7 & 90.2 \\
\hline ESO & $\mathbf{1 0 0}$ & $\mathbf{9 7 . 4}$ & $\mathbf{7 3 . 9}$ & $\mathbf{9 0 . 4}$ \\
\hline
\end{tabular}

tion variations is reported in Table 2. ESO performs substantially better than [8], and marginally better than [7, 9, 10]. Note that MFF [7], whose performance is very close to ESO, has more than 10 hyperparameters, causing difficulties for optimal parameter selection. In contrast, ESO has only 4 hyperparameters.

Runtime The optimisation time was measured on a computer with Intel Core2 Duo E8400 CPU and 4GB RAM memory. The results obtained for our implementation of the SimOpt method (MFF [7]) and the results reported for the SeqOpt method [10] are compared with those obtained with ESO. MFF took 23.1 seconds to fit one image, while ESO took only 2.1 seconds on average per fitting. The authors of [10] did not report their run time, but they also determined the albedo estimation to be the dominant step, with the same complexity of $O\left(n r_{t}^{2}\right)$. Note however that [10] uses not only one group of global $\boldsymbol{\alpha}$ and $\boldsymbol{\beta}$ but also four additional local groups to represent a face, while we only use the global parameters. Therefore $r_{t}$ in our approach is one fifth of [10], giving a 25 -fold speed advantage.

\subsubsection{Multi-PIE Database}

To compare with other state-of-the-art methods, evaluations are also conducted on a larger database, Multi-PIE, containing more than 750,000 images of 337 people. In addition, our face recognition systems, initialised by both manually and automatically detected landmarks, are compared. We used a cascaded regression-based automatic landmark detection method [25].

Protocol There are two settings, Setting-I and Setting-II, widely used in previous work $[11,12,36,38]$. Setting-I is used for face recognition in the presence of combined pose 
Table 3: Face recognition rate (\%) on different poses averaging all the illuminations on Multi-PIE (Setting-I)

\begin{tabular}{|c|c|c|c|c|c|c|c|c|c|c|}
\hline Method & Annotation & Feature & $-45^{\circ}$ & $-30^{\circ}$ & $-15^{\circ}$ & $+15^{\circ}$ & $+30^{\circ}$ & $+45^{\circ}$ & Mean & $0^{\circ}$ \\
\hline $\mathrm{Li}[31$ & Manual & Gabor & 63.5 & 69.3 & 79.7 & 75.6 & 71.6 & 54.6 & 69.1 & N/A \\
\hline \multirow{3}{*}{$\begin{array}{l}\text { Deep } \\
\text { Learning }\end{array}$} & \multirow{3}{*}{ Automatic } & RL [11] & 66.1 & 78.9 & 91.4 & 90.0 & 82.5 & 62.0 & 78.5 & 94.3 \\
\hline & & FIP 111 & 63.6 & 77.5 & 90.5 & 89.8 & 80.0 & 59.5 & 76.81 & 94.3 \\
\hline & & MVP 12 & 75.2 & 83.4 & 93.3 & 92.2 & 83.9 & 70.6 & 83.1 & 95.7 \\
\hline \multirow{4}{*}{ ESO } & \multirow{2}{*}{ Automatic } & Holistic & 73.8 & 87.5 & 95.0 & 95.1 & 90.0 & 76.2 & 86.3 & 98.7 \\
\hline & & Local & 79.6 & 91.6 & 98.2 & 97.9 & 92.6 & 81.3 & 90.2 & 99.4 \\
\hline & \multirow{2}{*}{ Manual } & Holistic & 80.8 & 88.9 & 96.7 & 97.6 & 93.3 & 81.1 & 89.7 & 99.1 \\
\hline & & Local & 81.1 & 93.3 & 97.7 & 98.0 & 93.3 & 82.4 & 91.0 & 99.6 \\
\hline
\end{tabular}

Table 4: Face recognition rate $(\%)$ on different poses under neutral illumination on Multi-PIE (Setting-II)

\begin{tabular}{|c|c|c|c|c|c|c|c|c|c|}
\hline & Method & Annotation & $-45^{\circ}$ & $-30^{\circ}$ & $-15^{\circ}$ & $+15^{\circ}$ & $+30^{\circ}$ & $+45^{\circ}$ & Mean \\
\hline \multirow{5}{*}{$2 \mathrm{D}$} & PLS 32] & \multirow{3}{*}{ Manual } & 51.1 & 76.9 & 88.3 & 88.3 & 78.5 & 56.5 & 73.3 \\
\hline & CCA 47 & & 53.3 & 74.2 & 90.0 & 90.0 & 85.5 & 48.2 & 73.5 \\
\hline & GMA 48 & & 75.0 & 74.5 & 82.7 & 92.6 & 87.5 & 65.2 & 79.6 \\
\hline & DAE 49 & \multirow{2}{*}{ Automatic } & 69.9 & 81.2 & 91.0 & 91.9 & 86.5 & 74.3 & 82.5 \\
\hline & SPAE [36 & & 84.9 & 92.6 & 96.3 & 95.7 & 94.3 & 84.4 & 91.4 \\
\hline \multirow{3}{*}{$3 \mathrm{D}$} & Asthana 38 & \multirow{3}{*}{ Automatic } & 74.1 & 91.0 & 95.7 & 95.7 & 89.5 & 74.8 & 86.8 \\
\hline & MDF 50 & & 78.7 & 94.0 & 99.0 & 98.7 & 92.2 & 81.8 & 90.7 \\
\hline & ESO+LPQ & & 91.7 & 95.3 & 96.3 & 96.7 & 95.3 & 90.3 & 94.4 \\
\hline
\end{tabular}

and illumination variations, Setting-II for that with only pose variations.

In common with [11, 12], Setting-I uses a subset in session 01 consisting of 249 subjects with 7 poses and 20 illumination variations. The images of the first 100 subjects constitute the training set. The remaining 149 subjects form the test set. In the test set, the frontal images under neutral illumination work as the gallery and the remaining are probe images. Following [36, 38], Setting-II uses the images of all the 4 sessions (01-04) under 7 poses and only neutral illumination. The images from the first 200 subjects are used for training and the remaining 137 subjects for testing. In the test set, the frontal images from session 01 work as gallery, and the others are probes.

ESO vs Deep Learning (Setting-I) In recent years, deep learning methods have achieved considerable success in a range of vision applications. In particular, deep learning works well for pose- and illumination-invariant face recognition [11, 12]. To our 
knowledge, these methods have reported the best face recognition rate so far on MultiPIE over both pose and illumination variations. Systems deploying these methods learned 3 pose- and illumination-invariant features: FIP (face identity-preserving), RL (FIP reconstructed features), and MVP (multi-view perceptron) using convolutional neural networks $(\mathrm{CNN})$. Table 3 compares ESO with these deep learning methods and the baseline method [31]. Not surprisingly, deep learning methods work better than [31] because of their powerful feature learning capability. However, ESO with automatic annotation, using either holistic or local features, outperforms these three deep learning solutions as shown in Table 3 We conclude that the superior performance of ESO results from the fact that the fitting process of ESO can explicitly model the pose. In contrast, the deep learning methods try to learn the view/pose-invariant features across different poses. This learning objective is highly non-linear so that the methods tend to get trapped in local minima. In contrast, ESO solves several convex problems and avoids this pitfall.

Automatic vs Manual Annotation (Setting-I) Table 3 also compares the performance of ESO with fully automatic annotation against that based on manual annotation. This table shows that the mean face recognition rates of the fully automatic system are close to those relying on manual annotation: $88.0 \%$ vs $91.2 \%$ for holistic features, and $91.5 \%$ vs $92.2 \%$ for local features. It means that ESO is reasonably robust to the errors caused by automatically detected landmarks.The superiority of local features, which can capture more facial details than holistic features, is also evident from the results.

ESO for Pose-robust Face Recognition (Setting-II) Table 4 compares ESO with the state-of-the-art methods for pose-robust face recognition. The methods can be classified into 2D and 3D approaches as discussed in Section 5.2 In the 2D category, PLS [32] and CCA [47] are unsupervised methods, and consequently they deliver inferior performance. GMA [48] benefits from its use of some additional supervisory information. DAE [49] and SPAE [36] are auto-encoder-based methods, which have superior capability to learn the non-linear relationships between images of different poses. SPAE set the state-of-the-art in performance, even compared with 3D methods [38] and [50]. However, our ESO outperforms SPAE, specifically $94.4 \%$ vs $91.4 \%$, 
because of its accurate shape and albedo reconstruction capability.

\section{Conclusions}

We proposed a new optimisation method - Efficient Stepwise Optimisation (ESO) - for fitting a 3D morphable face model to a 2D face image. In order to improve the optimisation efficiency, the method decouples the geometric and photometric optimisations and uses least squares sequentially to optimise the reconstructed shape, light direction, light strength and albedo parameters in separate steps. It includes a perspective camera model that becomes important in view of the growing interest in near-camera applications.

The computational efficiency of ESO is achieved thanks to the proposed linearisation of the model fitting steps, leading to closed-form solutions. ESO improves the optimisation efficiency by an order of magnitude in comparison with [7]. Moreover, it overcomes the weaknesses of earlier SeqOpt methods:

- The shape reconstruction of ESO supports a perspective camera.

- ESO linearises the Phong model.

- It models specularity.

- Occluding contour landmarks (Section 4.3) are used for a more robust fitting.

The experimental results demonstrate that the face reconstruction achievable by ESO is an improvement on that obtained from the state-of-the-art methods.

The ESO fitting algorithm can extract both holistic features and local features. A face recognition system that incorporates ESO to facilitate pose and illumination invariance was constructed, and evaluated on the PIE and Multi-Pie benchmark datasets with very promising results.

\section{Acknowledgments}

Support for this work is gratefully acknowledged from: EPSRC/DSTL project EP/K014307/1 "Signal processing in a networked battlespace"; EPSRC Programme 
Grant EP/L000539 "S3A: Future spatial audio for immersive listener experiences at home"; and the European Commission FP7 project 284989 "BEAT".

\section{References}

[1] R. Ramamoorthi, P. Hanrahan, A signal-processing framework for inverse rendering, in: Proceedings of the 28th annual conference on Computer graphics and interactive techniques, ACM, 2001, pp. 117-128.

[2] V. Blanz, T. Vetter, Face recognition based on fitting a 3D morphable model, Pattern Analysis and Machine Intelligence, IEEE Transactions on 25 (9) (2003) 1063-1074.

[3] X. Bai, E. R. Hancock, R. C. Wilson, A generative model for graph matching and embedding, Computer Vision and Image Understanding 113 (7) (2009) 777-789.

[4] X. Bai, E. R. Hancock, R. C. Wilson, Graph characteristics from the heat kernel trace, Pattern Recognition 42 (11) (2009) 2589-2606.

[5] V. Blanz, T. Vetter, A morphable model for the synthesis of 3D faces, in: Proceedings of the 26th annual conference on Computer graphics and interactive techniques, 1999, pp. 187-194.

[6] S. Romdhani, T. Vetter, Efficient, robust and accurate fitting of a 3D morphable model, in: ICCV, IEEE, 2003, pp. 59-66.

[7] S. Romdhani, T. Vetter, Estimating 3D shape and texture using pixel intensity, edges, specular highlights, texture constraints and a prior, in: CVPR, IEEE, 2005, pp. 986-993.

[8] S. Romdhani, V. Blanz, T. Vetter, Face identification by fitting a 3D morphable model using linear shape and texture error functions, in: ECCV, Springer, 2002, pp. 3-19.

[9] L. Zhang, D. Samaras, Face recognition from a single training image under arbitrary unknown lighting using spherical harmonics, Pattern Analysis and Machine Intelligence, IEEE Transactions on 28 (3) (2006) 351-363. 
[10] O. Aldrian, W. A. Smith, Inverse rendering of faces with a 3D morphable model, Pattern Analysis and Machine Intelligence, IEEE Transactions on 35 (5) (2013) 1080-1093.

[11] Z. Zhu, P. Luo, X. Wang, X. Tang, Deep learning identity preserving face space, in: Proc. ICCV, Vol. 1, 2013, p. 2.

[12] Z. Zhu, P. Luo, X. Wang, X. Tang, Deep learning multi-view representation for face recognition, arXiv preprint arXiv:1406.6947.

[13] C. P. Huynh, A. Robles-Kelly, E. R. Hancock, Shape and refractive index from single-view spectro-polarimetric images, International Journal of Computer Vision 101 (1) (2013) 64-94.

[14] R. Ramamoorthi, P. Hanrahan, A signal-processing framework for reflection, ACM Transactions on Graphics (TOG) 23 (4) (2004) 1004-1042.

[15] G. Hu, P. Mortazavian, J. Kittler, W. Christmas, A facial symmetry prior for improved illumination fitting of 3D morphable model, in: International Conference on Biometrics, IEEE, 2013, pp. 1-6.

[16] G. Hu, C. Chan, J. Kittler, W. Christmas, Resolution-aware 3D morphable model, in: British Machine Vision Conference, 2012, pp. 1-10.

[17] Y. Wang, Z. Liu, G. Hua, Z. Wen, Z. Zhang, D. Samaras, Face re-lighting from a single image under harsh lighting conditions, in: Computer Vision and Pattern Recognition, IEEE Conference on, IEEE, 2007.

[18] X. Zhu, Z. Lei, J. Yan, D. Yi, S. Z. Li, High-fidelity pose and expression normalization for face recognition in the wild, in: Proceedings of the IEEE Conference on Computer Vision and Pattern Recognition, 2015, pp. 787-796.

[19] X. Zhu, J. Yan, D. Yi, Z. Lei, S. Z. Li, Discriminative 3D morphable model fitting, in: Automatic Face and Gesture Recognition (FG), IEEE International Conference on, 2015. 
[20] A. Patel, W. A. Smith, 3D morphable face models revisited, in: CVPR, IEEE, 2009, pp. 1327-1334.

[21] P. Huber, Z. Feng, W. Christmas, J. Kittler, M. Rätsch, Fitting 3D Morphable Models using local features, in: IEEE International Conference on Image Processing, (ICIP), 2015. doi:10.1109/ICIP.2015.7350989.

URL http://dx.doi.org/10.1109/ICIP.2015.7350989

[22] W. A. P. Smith, E. R. Hancock, Estimating facial reflectance properties using shape-from-shading, International Journal of Computer Vision 86 (2-3) (2010) $152-170$.

[23] J. T. Rodriguez, $3 \mathrm{D}$ face modelling for $2 \mathrm{D}+3 \mathrm{D}$ face recognition $\mathrm{Ph} . \mathrm{D}$. thesis, Surrey University, Guildford, UK (2007).

URL http://wWw.ee.surrey.ac.uk/CVSSP/Publications/ papers/tena-2007.pdf

[24] A. Bas, W. A. P. Smith, T. Bolkart, S. Wuhrer, Fitting a 3D morphable model to edges: A comparison between hard and soft correspondences, CoRR abs/1602.01125.

URL http://arxiv.org/abs/1602.01125

[25] Z.-H. Feng, P. Huber, J. Kittler, W. Christmas, X.-J. Wu, Random cascadedregression copse for robust facial landmark detection, Signal Processing Letters, IEEE 22 (1) (2015) 76-80.

[26] I. Kemelmacher-Shlizerman, R. Basri, 3D face reconstruction from a single image using a single reference face shape, Pattern Analysis and Machine Intelligence, IEEE Transactions on 33 (2) (2011) 394-405.

[27] S. R. Marschner, S. H. Westin, E. P. Lafortune, K. E. Torrance, D. P. Greenberg, Image-based brdf measurement including human skin, in: Rendering Techniques 99, Springer, 1999, pp. 131-144.

[28] R. Hartley, A. Zisserman, Multiple view geometry in computer vision, Cambridge university press, 2003. 
[29] C. Ding, D. Tao, A comprehensive survey on pose-invariant face recognition, arXiv preprint arXiv:1502.04383.

[30] S. R. Arashloo, J. Kittler, Energy normalization for pose-invariant face recognition based on mrf model image matching, Pattern Analysis and Machine Intelligence, IEEE Transactions on 33 (6) (2011) 1274-1280.

[31] A. Li, S. Shan, W. Gao, Coupled bias-variance tradeoff for cross-pose face recognition, Image Processing, IEEE Transactions on 21 (1) (2012) 305-315.

[32] A. Sharma, D. W. Jacobs, Bypassing synthesis: PLS for face recognition with pose, low-resolution and sketch, in: CVPR, IEEE, 2011, pp. 593-600.

[33] A. B. Ashraf, S. Lucey, T. Chen, Learning patch correspondences for improved viewpoint invariant face recognition, in: CVPR, IEEE, 2008, pp. 1-8.

[34] T.-K. Kim, J. Kittler, R. Cipolla, Discriminative learning and recognition of image set classes using canonical correlations, Pattern Analysis and Machine Intelligence, IEEE Transactions on 29 (6) (2007) 1005-1018.

[35] S. J. Prince, J. Warrell, J. H. Elder, F. M. Felisberti, Tied factor analysis for face recognition across large pose differences, Pattern Analysis and Machine Intelligence, IEEE Transactions on 30 (6) (2008) 970-984.

[36] M. Kan, S. Shan, H. Chang, X. Chen, Stacked progressive auto-encoders (spae) for face recognition across poses, in: Proceedings of the IEEE Conference on Computer Vision and Pattern Recognition, 2013, pp. 1883-1890.

[37] C. Ding, C. Xu, D. Tao, Multi-task pose-invariant face recognition, Image Processing, IEEE Transactions on 24 (3) (2015) 980-993.

[38] A. Asthana, T. K. Marks, M. J. Jones, K. H. Tieu, M. Rohith, Fully automatic pose-invariant face recognition via 3D pose normalization, in: Computer Vision, International Conference on, IEEE, 2011, pp. 937-944. 
[39] R. Abiantun, U. Prabhu, M. Savvides, Sparse feature extraction for pose-tolerant face recognition, Pattern Analysis and Machine Intelligence, IEEE Transactions on 36 (10) (2014) 2061-2073.

[40] K. Niinuma, H. Han, A. K. Jain, Automatic multi-view face recognition via 3D model based pose regularization, in: Biometrics: Theory, Applications and Systems (BTAS), IEEE Conference on, 2013.

[41] U. Prabhu, J. Heo, M. Savvides, Unconstrained pose-invariant face recognition using 3D generic elastic models, Pattern Analysis and Machine Intelligence, IEEE Transactions on 33 (10) (2011) 1952-1961.

[42] D. Yi, Z. Lei, S. Z. Li, Towards pose robust face recognition, in: CVPR, IEEE, 2013, pp. 3539-3545.

[43] S. Schönborn, A. Forster, B. Egger, T. Vetter, A monte carlo strategy to integrate detection and model-based face analysis, in: Pattern Recognition, 2013.

[44] T. Ahonen, E. Rahtu, V. Ojansivu, J. Heikkila, Recognition of blurred faces using local phase quantization, in: ICPR, IEEE, 2008.

[45] T. Sim, S. Baker, M. Bsat, The CMU pose, illumination, and expression (PIE) database, in: Automatic Face and Gesture Recognition, IEEE International Conference on, 2002, pp. 46-51.

[46] R. Gross, I. Matthews, J. Cohn, T. Kanade, S. Baker, Multi-PIE, Image and Vision Computing 28 (5) (2010) 807-813.

[47] H. Hotelling, Relations between two sets of variates, Biometrika (1936) 321-377.

[48] A. Sharma, A. Kumar, H. Daume, D. W. Jacobs, Generalized multiview analysis: A discriminative latent space, in: CVPR, IEEE, 2012, pp. 2160-2167.

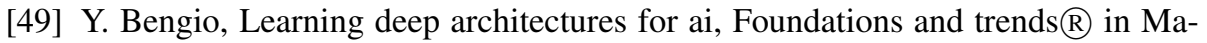
chine Learning 2 (1) (2009) 1-127. 
[50] S. Li, X. Liu, X. Chai, H. Zhang, S. Lao, S. Shan, Morphable displacement field based image matching for face recognition across pose, in: ECCV, Springer, 2012. 